\title{
Biocontrol in practice in Canadian floricultural greenhouses
}

\author{
Poonam Singh ${ }^{1 *}\left(\mathbb{D}\right.$, Tiffany Nykolyshyn ${ }^{1}(\mathbb{0}$ \\ ${ }^{1}$ Assiniboine Community College, School of Agriculture and Environment, Brandon-Manitoba, Canada.
}

\begin{abstract}
The environment inside Canadian prairie greenhouses differs from greenhouses built in other northern latitude locations in terms of lighting, temperature, humidity, and photoperiod. Since the performance of biocontrol agents depends upon several interactive environmental variables, their effectiveness to control pests in a particular crop growing under certain climatic conditions does not directly translate to another crop or location. So, we analyzed research trials assessing the efficacy and compatibility of various biocontrol agents (Amblyseius cucumeris, Amblyseius cucumeris, Phytoseiulus persimilis, Encarsia formosa, Aphidius colemani, Aphidius ervi, and Steinernema feltiae) on key pests (Western flower thrips, two-spotted spider mites, greenhouse whiteflies, and aphids) of spring bedding plants grown in a commercial floricultural greenhouse. Were analyzed several compatible combinations of biocontrol agents and observed a significant reduction in pest densities and plant damage symptoms as compared to untreated control plants. The results demonstrate that $P$. persimilis controlled two-spotted spider mites successfully in calibrachoa crop. The combination of Amblyseius cucumeris and $S$. feltiae resulted in significantly better control of Western flower thrips than the use of Amblyseius cucumeris alone in sweet potato vine plants. The application of E. formosa and Amblyseius cucumeris individually reduced greenhouse whiteflies on calibrachoa plants as compared to control, but their combination performed better resulting in a significantly lower number of whiteflies on plants. Another combination of Aphidius colemani and Aphidius ervi controlled green peach aphids and foxglove aphids effectively on the pansy crop. The biocontrol agents were effective for managing a variety of pests in a commercial greenhouse setting.
\end{abstract}

Keywords: biocontrol agents, controlled environment, pests, spring bedding plants.

\section{Resumo}

\section{Biocontrole na prática em estufas canadenses de ornamentais}

O ambiente interno das estufas das pradarias canadenses difere das estufas construídas em outros locais da latitude norte em termos de iluminação, temperatura, umidade e fotoperíodo. Uma vez que o desempenho dos agentes de biocontrole depende da interação de algumas variáveis ambientais, sua eficácia no controle de pragas em uma cultura específica que está crescendo sob certas condições climáticas não se traduz diretamente em outras plantas ou local. Portanto, foram analisados ensaios de pesquisa que avaliaram a eficácia e compatibilidade de vários agentes de biocontrole (Amblyseius cucumeris, Amblyseius cucumeris, Phytoseiulus persimilis, Encarsia formosa, Aphidius colemani, Aphidius ervi, e Steinernema feltiae) sobre pragas principais (tripes de flores ocidentais, dois ácaros aranha-pintados, mosca-branca de estufa e pulgões) em plantas de forração para primavera cultivadas em estufa de floricultura comercial. Foram analisadas várias combinações compatíveis de agentes de biocontrole e observou-se uma redução significativa nas densidades de pragas e sintomas de danos às plantas em comparação com plantas controle não tratadas. Os resultados demonstram que P. persimilis controlou com sucesso os ácaros aranha-pintados na cultura da calibrachoa. A combinação de Amblyseius cucumeris e $S$. feltiae resultou em um controle significativamente melhor de tripes de flores ocidentais do que somente o uso de Amblyseius cucumeris em plantas de videira de batata-doce ornamental. A aplicação de E. formosa e Amblyseius cucumeris reduziu individualmente as moscas-brancas em plantas de calibrachoa em comparação com o controle, mas sua combinação apresentou melhor desempenho, resultando em um número significativamente menor de moscas-brancas nas plantas. A combinação de Aphidius colemani e Aphidius ervi controlou efetivamente pulgões verdes e pulgões dedaleira na cultura do amor-perfeito. Os agentes de controle biológico foram eficazes no manejo de uma variedade de pragas em uma estufa comercial de ornamentais.

Palavras-chave: agentes de biocontrole, ambiente controlado, pragas, forrações para primavera

\footnotetext{
https://doi.org/10.1590/2447-536X.v27i4.2354
}

*Corresponing author: singhp@assiniboine.net 


\section{Introduction}

Canadian Prairie Provinces receive ample amounts of bright sunlight and longer day lengths as compared to other parts of the country where the greenhouse industry is mostly concentrated. Most prairie greenhouses use air inflated double polyethylene (due to continuous freezing and thawing of the ground) as covering material in contrast to glass, which is a material of choice in most other greenhouse growing regions. Due to these factors, the environmental conditions inside the greenhouses situated on the Canadian prairies are different from those located in other parts of Canada or Europe.

Canadian floriculture greenhouses produce a wide variety of crops, including bedding plants, cut flowers, perennials, and propagation material. Crops are produced on a large scale, with high species diversity in highdensity greenhouse plantings. With diversity comes complications, such as ever-changing crop mixes, plant species susceptibility to various pests, the introduction of new crops that are potential hosts to pests, and high-quality standards imposed by the customers. Due to the importance of aesthetic quality of ornamental plants, even very small damage symptoms on leaves and petals are regarded as a reduction in the quality of these plants (Alipour et al., 2019). Plants arrive regularly from many different sources increasing the chances of pest introductions. There is also a continuous movement of plants from one growing site to another throughout the production cycle. Retail greenhouses, being open to the public, see variations in the sales of different plants; some plants sell quickly while others stay for relatively long periods. A longer stay increases the chances for pest incidence and development.

The susceptibility of plants to pests and the performance of biocontrol agents in the greenhouse depends on several environmental conditions like temperature, humidity, and sunlight (Brownbridge, 2017). Understanding how environmental factors interact with and affect the plants, pests, and their biocontrol agents, is essential for implementing a successful biological pest control program in the greenhouses, thus preventing a 'one-size-fits-all' approach. Most Canadian flower growers feel that the lack of connection to research or research into biological control options is one of the limiting factors in achieving adequate management of changing pest threats (Summerfield, 2019).

Our preliminary studies carried out in 2015-16 at the Assiniboine Community Colleges' (ACC) sustainable greenhouse located in Brandon, Manitoba revealed that the biocontrol agents were effective in significantly reducing the pest populations and damage to several ornamental and food crops. In the present study, we focused on key greenhouse pests viz. Western flower thrips Frankliniella occidentalis Pergande (Thysanoptera: Thripidae), Greenhouse whiteflies Trialeurodes vaporariorum Westwood (Hemiptera: Aleyrodidae), Green peach aphids Myzus persicae Sulzer (Hemiptera: Aphididae), Foxglove aphids Aulacorthum solani Kaltenbach (Hemiptera: Aphididae), and two-spotted spider mites Tetranychus urticae Koch (Acari: Tetranychidae), commonly found in commercial greenhouse environments. These pests were chosen based on their high prevalence in the greenhouse as suggested by the greenhouse growers. Most of these pests were poorly controlled by chemical pesticides and had resulted in major losses of the crop in the past years. There are several biocontrol agents available in the market that are recommended by companies to use on an individual basis. However, many of these could work synergistically and could be effective for managing more than single pest species (Garriga et al., 2019). In general, there is a lack of scientific papers that deal with the outcome of biological control at field or greenhouse scale, as too often early lab trials are documented, while full-scale results stay with companies or growers. Therefore, the objective of the study was to assess the efficacy and compatibility of several biocontrol agents in a commercial floricultural greenhouse setting.

\section{Materials and Methods}

The experiments were conducted during the springsummer season of years, 2017 and 2018 at Shelmerdine Garden Centre Ltd. located at Headingley, Manitoba, Canada $\left(49^{\circ} 51^{\prime} \mathrm{N}, 97^{\circ} 22^{\prime} \mathrm{W}, 238\right.$ meters above the sea level). The average climatic parameters from 1981-2015 for the Winnipeg region are as follows: mean temperature varied from $-13{ }^{\circ} \mathrm{C}$ to $25{ }^{\circ} \mathrm{C}$; the frost-free days were in the range of 125 to 135 . The region received annual sunshine hours of 2353; daylight hours ranged from 12 hours 53 minutes in March, 14 hours 40 minutes in April, 15 hours 30 minutes in May, 16 hours 02 minutes in June, 16 hours and 15 minutes in July, 15 hours and 10 minutes in August, and 14 hours 10 minutes in September. The average precipitation that the region received between May and September months was $389 \mathrm{~mm}$. The average annual relative humidity was $69.6 \%$ and monthly average relative humidity ranged from $49 \%$ in May to $87 \%$ in February (Environmental Canada Weather Station ECCC-MSC, Winnipeg).

Shelmerdine, the largest retail garden center in the province of Manitoba, had a gutter-connected greenhouse that was made up of aluminum and the covering material was composed of double-layer polycarbonate in the retail area, and air-inflated double polyethylene in the production area. Partitions within the greenhouse were created by erecting polycarbonate walls connected through the sliding doors, dividing the production area into 5 zones. The ebb and flow system of irrigation was used for irrigating bedding plant crops. The drip system of irrigation was used for the hanging baskets. The use of fertilizer largely depended upon the type, requirement, and growth stage of the crop, but in general, the fertilizer applied in the greenhouse were 15-0-15 and 20-8-20+micros. The mean air temperature fluctuated between $17.0^{\circ} \mathrm{C}$ and $38.5^{\circ} \mathrm{C}$ and the mean soil temperature ranged between $15.7{ }^{\circ} \mathrm{C}$ and $38.3{ }^{\circ} \mathrm{C}$ during the study period. The biocontrol agents were procured from Biobest Canada Ltd. through a local distributor, EvanSpray \& Chemicals Ltd., Winnipeg, Manitoba, Canada. The biocontrol agents used in the study include predatory 
mites, viz. Amblyseius cucumeris Oudemans (Acari: Phytoseiidae), Amblyseius swirskii Athias-henriot (Acari: Phytoseiidae), Phytoseiulus persimilis Athias-Henriot (Acari: Phytoseiidae); parasitoid wasps viz. Encarsia formosa Gahan (Hymenoptera: Aphelinidae), Aphidius colemani Viereck (Hymenoptera: Braconidae), Aphidius ervi Haliday (Hymenoptera: Braconidae); and parasitic nematode, Steinernema feltiae Filipjev (Rhabditida: Steinernematidae) as biocontrol agents against the pests mentioned above.

Data analysis was conducted using analyses of variance (ANOVA) with CoStat ver. 6.45 (CoHort Software, USA). Differences between treatment means ( \pm standard deviation) were determined using Fisher's least significant difference at $\mathrm{P}=5 \%$. The experimental set up is described below:

Experiment 1: Efficacy of $P$. persimilis for the control of two-spotted spider mites (TSSM), T. urticae, on calibrachoa (Calibrachoa $x$ hybrida 'Conga'TM Deep Yellow').

Fifty plants of Calibrachoa $x$ hybrida 'Conga ${ }^{\mathrm{TM}}$ Deep Yellow' planted in 8-inch pots were randomly selected for this experiment. The plants were at the vegetative stage for the first 7 weeks of study and were at the flowering stage thereafter (Table 1).

Table 1. Effect of P. persimilis on two-spotted spider mites (T. urticae) in calibrachoa

\begin{tabular}{|c|c|c|c|c|c|}
\hline & \multirow{2}{*}{ Plant Growth Stage } & \multicolumn{2}{|c|}{${ }^{1} P$. persimilis } & \multicolumn{2}{|c|}{${ }^{2}$ Control } \\
\hline & & $\begin{array}{c}* * \text { TSSM per } \\
\text { plant }\end{array}$ & $\begin{array}{c}* * * \text { Plant Damage } \\
\text { Rating }\end{array}$ & $\begin{array}{c}* * \text { TSSM per } \\
\text { plant }\end{array}$ & $\begin{array}{c}* * * \text { Plant Damage } \\
\text { Rating }\end{array}$ \\
\hline Week 1 & 8-10 leaves & $0.40 \pm 0.20 \mathrm{a}$ & $1.00 \mathrm{a}$ & $0.60 \pm 0.20 \mathrm{a}$ & $1.00 \mathrm{a}$ \\
\hline Week 2 & 10-12 leaves & $0.60 \pm 0.22 \mathrm{a}$ & $1.00 \mathrm{a}$ & $1.00 \pm 0.52 \mathrm{a}$ & $1.00 \mathrm{a}$ \\
\hline Week 3 & 10-12 leaves & $0.80 \pm 0.10 \mathrm{a}$ & $1.00 \mathrm{a}$ & $0.60 \pm 0.31 \mathrm{a}$ & $1.00 \mathrm{a}$ \\
\hline Week 4 & 4-6 laterals & $4.40 \pm 1.56 \mathrm{a}$ & $2.00 \mathrm{a}$ & $5.00 \pm 2.32 \mathrm{a}$ & $2.00 \mathrm{a}$ \\
\hline Week 5 & 6-10 laterals & $6.20 \pm 2.45 \mathrm{a}$ & $2.00 \mathrm{~b}$ & $6.60 \pm 3.00 \mathrm{a}$ & $3.00 \mathrm{a}$ \\
\hline *Week 6 & $\begin{array}{l}\text { 8-10 laterals, } 16-18 \\
\text { secondary laterals }\end{array}$ & $16.80 \pm 3.33 \mathrm{a}$ & $2.00 \mathrm{~b}$ & $15.00 \pm 5.21 \mathrm{a}$ & $3.00 \mathrm{a}$ \\
\hline *Week 7 & $\begin{array}{l}\text { 8-10 laterals, } 16-18 \\
\text { secondary laterals }\end{array}$ & $16.00 \pm 4.30 \mathrm{a}$ & $3.00 \mathrm{a}$ & $17.20 \pm 4.88 \mathrm{a}$ & $3.00 \mathrm{a}$ \\
\hline Week 8 & Flowering & $13.20 \pm 2.67 b$ & $3.00 \mathrm{~b}$ & $18.20 \pm 6.77 \mathrm{a}$ & $4.00 \mathrm{a}$ \\
\hline Week 9 & Flowering & $5.20 \pm 2.00 \mathrm{~b}$ & $2.00 \mathrm{~b}$ & $25.00 \pm 6.77 \mathrm{a}$ & $4.00 \mathrm{a}$ \\
\hline Week 10 & Flowering & $4.00 \pm 1.00 \mathrm{~b}$ & $2.00 \mathrm{~b}$ & $35.00 \pm 7.67 \mathrm{a}$ & $5.00 \mathrm{a}$ \\
\hline Week 11 & Flowering & $4.20 \pm 2.80 \mathrm{~b}$ & $2.00 \mathrm{~b}$ & $40.00 \pm 8.98 \mathrm{a}$ & $5.00 \mathrm{a}$ \\
\hline Week 12 & Flowering & $3.80 \pm 2.33 b$ & $3.00 \mathrm{~b}$ & $56.20 \pm 9.89 \mathrm{a}$ & $6.00 \mathrm{a}$ \\
\hline Week 13 & Flowering & $4.00 \pm 2.22 b$ & $4.00 \mathrm{~b}$ & $78.00 \pm 10.09 a$ & $6.00 \mathrm{a}$ \\
\hline Week 14 & Flowering & $6.20 \pm 1.98 b$ & $4.00 \mathrm{~b}$ & $99.80 \pm 15.67 \mathrm{a}$ & $6.00 \mathrm{a}$ \\
\hline *Week 15 & Flowering & $12.00 \pm 4.30 \mathrm{~b}$ & $4.00 \mathrm{~b}$ & $102.20 \pm 20.33 \mathrm{a}$ & $7.00 \mathrm{a}$ \\
\hline *Week 16 & Flowering & $11.80 \pm 3.34 \mathrm{~b}$ & $4.00 \mathrm{~b}$ & $110.00 \pm 24.54 \mathrm{a}$ & $8.00 \mathrm{a}$ \\
\hline Week 17 & Flowering & $7.20 \pm 2.33 b$ & $4.00 \mathrm{~b}$ & $120.60 \pm 24.00 \mathrm{a}$ & $9.00 \mathrm{a}$ \\
\hline Week 18 & Flowering & $7.20 \pm 2.00 \mathrm{~b}$ & $4.00 \mathrm{~b}$ & $134.40 \pm 36.66 a$ & $9.00 \mathrm{a}$ \\
\hline Week 19 & Flowering & $3.20 \pm 1.00 \mathrm{~b}$ & $4.00 \mathrm{~b}$ & $126.00 \pm 20.99 a$ & $9.00 \mathrm{a}$ \\
\hline Week 20 & Flowering & $2.80 \pm 1.34 \mathrm{~b}$ & $4.00 \mathrm{~b}$ & $144.40 \pm 40.00 \mathrm{a}$ & $9.00 \mathrm{a}$ \\
\hline
\end{tabular}

${ }^{1}$ P. persimilis $=8$ per $\mathrm{m}^{2}$ during $*$ weeks $6,7,15,16 ;{ }^{2}$ Control=Untreated plants

**Means represent 2-year averages of 25 plants per treatment ( 2 treatments, 5 replications, 5 plants per replication, 50 plants in total)

***Plant damage rating: $1-9$ scale (\% leaf area affected): $1=0-5,2=6-10,3=11-20,4=21-30,5=31-40,6=41-50,7=51-60,8=61-70,9=>70$

Number followed by the same letter are not significantly different in LSD at $\mathrm{P}=0.05$

The experiment was laid out in the Randomized Complete Block Design with 5 replications and 5 plants per replication. All 5 production zones received plants from each replicate of every treatment. Two random groups of 25 plants each were created: 1) $P$. persimilis treatment group, 2) untreated control group. Each group of plants was placed in $3 \mathrm{~m}^{2}$ space inside a cage built with plastic pipes and covered with tightly woven breathable sheer fabric that did not allow the escape of insects. Two treatments were separated with 30 meters of space. Cages were placed close to the rest of the crop plants. The environmental conditions inside the cages were the same as the rest of the greenhouse. 
P. persimilis mites mixed with bran were obtained in a sprinkle bottle delivery system. The mixture was spread evenly on the foliage of infested plants placed in close contact to promote dispersal of mites. The application was carried out at the rate of 8 mites per $\mathrm{m}^{2}$ as recommended by the manufacturer during weeks 6 and 7, and again during weeks 15 and 16. Two-spotted spider mites on the plants were scouted using a hand lens of focal distance $2.5 \mathrm{~cm}$, magnification $40 \mathrm{X}$, and diameter $25 \mathrm{~mm}$; every mite on all aboveground plant parts (leaves, stem, and flowers) was counted every week on the same day of the week, and averages were calculated. The initial number of TSSM at the start of monitoring (week 1) was less than 1 per plant. The damage symptoms on plants were recorded as percent leaf area affected using the following rating scale of 1 to 9: 1 (0-5\%), 2 (6\%-10\%), 3 (11\%-20\%), 4 (21\%-30\%), 5 (31\%-40\%), 6 (41\%-50\%), 7 (51\%-60\%) 8 (61\%-70\%), $9(>70 \%$, pronounced stunting of the plant, severe leaf shedding, plant death).

Experiment 2: Efficacy of Amblyseius cucumeris and $S$. feltiae for the control of western flower thrips (WFT), F. occidentalis, on sweet potato vine (Ipomoea batatas 'Marguerite').

A total of 75 randomly selected mature plants of sweet potato vine (I. batatas 'Marguerite') planted in 10-inch pots were divided into 3 treatment groups of 25 plants each. The treatment groups were: 1) Amblyseius cucumeris $+S$. feltiae, 2) Amblyseius cucumeris, and 3) untreated control. The selected plants were at the 8-10 leaves stage at the start of monitoring (week 1) and grew to have dense foliage trailing through the sides of the pots from week 8 onwards (Table 2).

The layout and setup of the experiment were the same as experiment 1. Amblyseius cucumeris was applied as slow-release sachets staked into the growing medium using bamboo sticks. There was a small opening on sachets on one side for the gradual dispersal of mites. Each sachet that was reported to last for 4 weeks contained 250 mites in a mix comprised of a food source for the reproduction of Amblyseius cucumeris. The release rate recommended by the manufacturer was 1 sachet per $\mathrm{m}^{2}$, but due to the low number of WTF on our plants, we decreased the application rate to 1 sachet in $3 \mathrm{~m}^{2}$ area. A package of $S$. feltiae containing 250 million nematodes was dissolved in 100 liters of water and applied at the rate of 500,000 nematodes per $\mathrm{m}^{2}$ (manufacturer's recommendation) by drenching growing media with the solution. A total of 5 applications (weeks 3, 7, 11, 15, and 19) of Amblyseius cucumeris and $S$. feltiae in a 20 -week production cycle were carried out. The damage symptoms on plants were recorded using a rating scale of 1 to 9 , the same as used in experiment 1 . Weekly data on the pest populations was recorded by counting all the WFT insects both on the plants and the yellow sticky cards (BUG-SCAN $\left.{ }^{\circledR}\right)$. Also, the foliage of each plant was shaken over a sheet of white paper and the number of dropped WFT (larvae and adults) were counted. These numbers were added to the WFT counts on the plants, and means were calculated. The yellow sticky cards cut into 4-inch squares were placed equidistantly among the crop; all WFT on both sides of the cards were counted and means were calculated. The initial number of WTF among treatments at the start of monitoring (week 1) was between 20.80 to 28.20 per plant and 113.40 to 130.00 per sticky card.

Experiment 3: Efficacy of E. formosa and Amblyseius cucumeris for the control of greenhouse whitefly (GW), T. vaporariorum on calibrachoa (Calibrachoa $x$ hybrida 'Chameleon ${ }^{\circledR}$ ').

The experiment was performed on calibrachoa (Calibrachoa $x$ hybrida 'Chameleon ${ }^{\circledR}$ ') planted in 8-inch pots. The plants were at the vegetative stage for the first 7 weeks of study and were at the flowering stage thereafter (Table 3). 
Table 2. Effect of Amblyseius cucumeris and S. feltiae on western flower thrips (F.occidentalis) in sweet potato vine

\begin{tabular}{|c|c|c|c|c|c|c|c|c|c|c|}
\hline & \multirow{2}{*}{$\begin{array}{c}\text { Plant } \\
\text { Growth } \\
\text { Stage }\end{array}$} & \multicolumn{3}{|c|}{${ }^{1}$ A.cucumeris + S.feltiae } & \multicolumn{3}{|c|}{${ }^{2}$ A.cucumeris } & \multicolumn{3}{|c|}{${ }^{3}$ Control } \\
\hline & & $\begin{array}{c}\text { **WFT (larvae } \\
+ \text { adults) per } \\
\text { plant }\end{array}$ & $\begin{array}{c}* * * \text { WFT (larvae + } \\
\text { adults) per sticky } \\
\text { card }\end{array}$ & $\begin{array}{l}{ }^{* * *} \text { Plant } \\
\text { Damage } \\
\text { Rating }\end{array}$ & $\begin{array}{l}* * \text { WFT (larvae }+ \\
\text { adults) per plant }\end{array}$ & $\begin{array}{c}\text { ***WFT (larvae } \\
\text { + adults) per } \\
\text { sticky card }\end{array}$ & $\begin{array}{l}{ }^{* * *} \text { Plant } \\
\text { Damage } \\
\text { Rating }\end{array}$ & $\begin{array}{l}\text { **WFT (larvae }+ \\
\text { adults) per plant }\end{array}$ & $\begin{array}{c}* * * \text { WFT (larvae }+ \\
\text { adults) per sticky } \\
\text { card }\end{array}$ & $\begin{array}{l}{ }^{* * *} \text { Plant } \\
\text { Damage } \\
\text { Rating }\end{array}$ \\
\hline Week 1 & 10-12 leaves & $28.20 \pm 9.83 a$ & $129.40 \pm 24.01 \mathrm{a}$ & $5.00 \mathrm{~b}$ & $20.80 \pm 10.00 \mathrm{~b}$ & $113.40 \pm 29.02 b$ & $5.00 \mathrm{a}$ & $25.60 \pm 13.33 \mathrm{ab}$ & $130.00 \pm 43.54 a$ & $6.00 \mathrm{a}$ \\
\hline Week 2 & $10-12$ leaves & $24.60 \pm 6.78 \mathrm{~b}$ & $120.00 \pm 30.00 \mathrm{~b}$ & $5.00 \mathrm{c}$ & $19.60 \pm 8.08 b$ & $88.40 \pm 12.22 \mathrm{c}$ & $5.00 \mathrm{~b}$ & $28.40 \pm 6.77 \mathrm{a}$ & $144.40 \pm 23.33 \mathrm{a}$ & $6.00 \mathrm{a}$ \\
\hline *Week 3 & 12-15 leaves & $14.20 \pm 4.03 \mathrm{~b}$ & $109.40 \pm 11.11 \mathrm{~b}$ & $5.00 \mathrm{c}$ & $14.40 \pm 7.80 \mathrm{~b}$ & $75.40 \pm 10.04 \mathrm{c}$ & $5.00 \mathrm{~b}$ & $32.00 \pm 9.89 \mathrm{a}$ & $150.00 \pm 33.33 \mathrm{a}$ & $6.00 \mathrm{a}$ \\
\hline Week 4 & 14-15 leaves & $12.80 \pm 5.78 \mathrm{~b}$ & $68.20 \pm 9.90 \mathrm{~b}$ & $4.00 \mathrm{~b}$ & $15.80 \pm 10.99 \mathrm{~b}$ & $55.60 \pm 16.76 \mathrm{c}$ & $5.00 \mathrm{a}$ & $56.80 \pm 11.11 \mathrm{a}$ & $170.20 \pm 49.90 \mathrm{a}$ & $6.00 \mathrm{a}$ \\
\hline Week 5 & 16-18 leaves & $4.20 \pm 1.98 c$ & $43.00 \pm 15.00 \mathrm{~b}$ & $4.00 \mathrm{~b}$ & $11.60 \pm 6.68 \mathrm{~b}$ & $30.60 \pm 8.08 \mathrm{c}$ & $3.00 \mathrm{~b}$ & $40.20 \pm 5.67 \mathrm{a}$ & $200.00 \pm 30.00 \mathrm{a}$ & $7.00 \mathrm{a}$ \\
\hline Week 6 & 16-18 leaves & $5.20 \pm 3.74 b$ & $32.20 \pm 10.00 \mathrm{~b}$ & $3.00 \mathrm{~b}$ & $7.20 \pm 4.00 \mathrm{~b}$ & $20.40 \pm 4.55 \mathrm{c}$ & $3.00 \mathrm{~b}$ & $45.60 \pm 12.32 \mathrm{a}$ & $211.40 \pm 68.90 \mathrm{a}$ & $7.00 \mathrm{a}$ \\
\hline *Week 7 & 16-18 leaves & $3.40 \pm 2.00 \mathrm{~b}$ & $20.00 \pm 8.88 b$ & $3.00 \mathrm{c}$ & $5.80 \pm 2.40 \mathrm{~b}$ & $25.20 \pm 9.87 b$ & $3.00 \mathrm{~b}$ & $50.00 \pm 7.78 \mathrm{a}$ & $220.00 \pm 35.60 \mathrm{a}$ & $7.00 \mathrm{a}$ \\
\hline Week 8 & 18-20 leaves & $1.80 \pm 0.90 \mathrm{~b}$ & $22.40 \pm 10.00 \mathrm{~b}$ & $3.00 \mathrm{c}$ & $4.20 \pm 3.00 \mathrm{~b}$ & $19.40 \pm 7.01 \mathrm{~b}$ & $3.00 \mathrm{~b}$ & $62.40 \pm 4.09 \mathrm{a}$ & $225.00 \pm 60.90 \mathrm{a}$ & $7.00 \mathrm{a}$ \\
\hline Week 9 & 18-20 leaves & $1.00 \pm 0.50 \mathrm{~b}$ & $18.80 \pm 6.67 \mathrm{~b}$ & $3.00 \mathrm{~b}$ & $6.20 \pm 3.59 b$ & $27.00 \pm 6.66 \mathrm{~b}$ & $3.00 \mathrm{~b}$ & $76.00 \pm 18.09 \mathrm{a}$ & $244.40 \pm 89.00 \mathrm{a}$ & $8.00 \mathrm{a}$ \\
\hline Week 10 & 18-20 leaves & $0.80 \pm 0.14 b$ & $8.80 \pm 3.90 \mathrm{~b}$ & $2.00 \mathrm{c}$ & $6.80 \pm 5.05 b$ & $20.00 \pm 7.08 b$ & $3.00 \mathrm{~b}$ & $78.00 \pm 7.86 a$ & $270.20 \pm 45.50 \mathrm{a}$ & $8.00 \mathrm{a}$ \\
\hline *Week 11 & 20-22 leaves & $1.20 \pm 1.00 \mathrm{~b}$ & $10.00 \pm 4.50 \mathrm{~b}$ & $2.00 \mathrm{~b}$ & $6.22 \pm 2.34 b$ & $17.60 \pm 10.09 b$ & $2.00 \mathrm{~b}$ & $82.20 \pm 10.02 \mathrm{a}$ & $280.20 \pm 67.78 \mathrm{a}$ & $8.00 \mathrm{a}$ \\
\hline Week 12 & 20-22 leaves & $5.20 \pm 2.56 b$ & $5.00 \pm 3.00 \mathrm{~b}$ & $2.00 \mathrm{c}$ & $6.80 \pm 3.45 b$ & $12.60 \pm 4.56 \mathrm{~b}$ & $3.00 \mathrm{~b}$ & $85.60 \pm 14.34 \mathrm{a}$ & $275.80 \pm 45.00 \mathrm{a}$ & $8.00 \mathrm{a}$ \\
\hline Week 13 & 20-22 leaves & $3.80 \pm 1.88 b$ & $2.20 \pm 1.20 \mathrm{~b}$ & $2.00 \mathrm{c}$ & $4.80 \pm 2.40 \mathrm{~b}$ & $15.20 \pm 6.89 \mathrm{~b}$ & $3.00 \mathrm{~b}$ & $90.00 \pm 17.87 \mathrm{a}$ & $288.40 \pm 66.90 \mathrm{a}$ & $8.00 \mathrm{a}$ \\
\hline Week 14 & 20-22 leaves & $2.40 \pm 1.90 \mathrm{~b}$ & $10.00 \pm 3.43 b$ & $2.00 \mathrm{c}$ & $6.10 \pm 4.04 \mathrm{~b}$ & $15.40 \pm 5.90 \mathrm{~b}$ & $2.00 \mathrm{~b}$ & $77.20 \pm 12.89 \mathrm{a}$ & $300.00 \pm 90.09 a$ & $8.00 \mathrm{a}$ \\
\hline *Week 15 & 24-26 leaves & $5.60 \pm 3.34 b$ & $10.80 \pm 5.55 b$ & $2.00 \mathrm{c}$ & $8.08 \pm 3.33 b$ & $10.20 \pm 6.76 \mathrm{~b}$ & $3.00 \mathrm{~b}$ & $82.20 \pm 17.89 a$ & $302.20 \pm 77.00 \mathrm{a}$ & $9.00 \mathrm{a}$ \\
\hline Week 16 & 24-26 leaves & $3.80 \pm 1.00 \mathrm{~b}$ & $2.00 \pm 1.00 \mathrm{~b}$ & $2.00 \mathrm{c}$ & $3.20 \pm 1.44 \mathrm{~b}$ & $8.80 \pm 5.65 b$ & $3.00 \mathrm{~b}$ & $88.80 \pm 16.89 a$ & $299.00 \pm 52.20 \mathrm{a}$ & $9.00 \mathrm{a}$ \\
\hline Week 17 & 24-26 leaves & $4.80 \pm 3.89 b$ & $4.00 \pm 3.40 \mathrm{~b}$ & $2.00 \mathrm{c}$ & $4.80 \pm 2.67 b$ & $14.00 \pm 4.40 \mathrm{~b}$ & $3.00 \mathrm{~b}$ & $89.60 \pm 15.55 \mathrm{a}$ & $324.80 \pm 89.98 \mathrm{a}$ & $9.00 \mathrm{a}$ \\
\hline Week 18 & 24-26 leaves & $1.00 \pm 0.20 \mathrm{~b}$ & $3.20 \pm 2.20 b$ & $2.00 \mathrm{c}$ & $6.80 \pm 2.88 \mathrm{~b}$ & $14.40 \pm 9.98 b$ & $3.00 \mathrm{~b}$ & $91.00 \pm 26.78 \mathrm{a}$ & $284.40 \pm 39-99 a$ & $9.00 \mathrm{a}$ \\
\hline *Week 19 & 24-26 leaves & $1.20 \pm 0.22 b$ & $3.00 \pm 1.23 b$ & $2.00 \mathrm{~b}$ & $6.50 \pm 1.78 b$ & $15.60 \pm 13.33 b$ & $3.00 \mathrm{~b}$ & $85.40 \pm 17.89 \mathrm{a}$ & $331.00 \pm 80.01 \mathrm{a}$ & $9.00 \mathrm{a}$ \\
\hline Week 20 & 24-26 leaves & $1.40 \pm 0.50 \mathrm{~b}$ & $1.30 \pm 0.42 b$ & $2.00 \mathrm{c}$ & $3.20 \pm 0.76 b$ & $13.00 \pm 8.00 \mathrm{~b}$ & $3.00 \mathrm{~b}$ & $106.00 \pm 43.00 \mathrm{a}$ & $334.40 \pm 55.23 \mathrm{a}$ & $9.00 \mathrm{a}$ \\
\hline
\end{tabular}

${ }^{1}$ Amblyseius cucumeris +S.feltiae treatment=Amblyseius cucumeris @1 sachet/3 $m^{2}+$ S. feltiae @ 1 million per 2m² during *weeks 3, 7,11,15,19

${ }^{2}$ Amblyseius cucumeris treatment @ 1 sachet $/ 3 m^{2}$ during *weeks 3, 7,11,15,19; ${ }^{3}$ Control=Untreated plants

"Means represent 2-year averages of 25 plants per treatment ( 3 treatments, 5 replications, 5 plants per replication, 75 plants in total)

${ }^{* * *}$ Means represent 2-year averages of 25 card counts per treatment ( 3 treatment, 5 replications, 5 cards per replication ( 75 cards in total)

***Plant damage rating: $1-9$ scale (\% leaf area affected): $1=0-5,2=6-10,3=11-20,4=21-30,5=31-40,6=41-50,7=51-60,8=61-70,9=>70$

Number followed by the same letter are not significantly different in LSD at $\mathrm{P}=0.05$ 
Table 3. Effect of E. formosa and Amblyseius cucumeris on greenhouse whitefly (T. vaporariorum) in calibrachoa

\begin{tabular}{|c|c|c|c|c|c|c|c|c|c|c|c|c|c|c|c|c|c|}
\hline & \multirow[b]{2}{*}{$\begin{array}{l}\text { Plant Growth } \\
\text { Stage }\end{array}$} & \multicolumn{4}{|c|}{${ }^{1}$ E. Formosa + A.swirskii } & \multicolumn{4}{|c|}{${ }^{2}$ A.swirskii } & \multicolumn{4}{|c|}{${ }^{3} E$. formosa } & \multicolumn{4}{|c|}{${ }^{4}$ Control } \\
\hline & & $\begin{array}{c}* \mathrm{GW} \text { adults } \\
\text { /plant }\end{array}$ & $\begin{array}{c}{ }^{*} \mathrm{GW} \\
\text { nymphs / } \\
\text { plant }\end{array}$ & $\begin{array}{l}\text { *Parasitized } \\
\text { GW nymphs } \\
\text { /plant }\end{array}$ & $\begin{array}{c}* * \text { Plant } \\
\text { damage } \\
\text { rating }\end{array}$ & $\begin{array}{c}* \text { GW adults } \\
\text { /plant }\end{array}$ & $\begin{array}{l}\text { *GW } \\
\text { nymphs/ } \\
\text { plant }\end{array}$ & $\begin{array}{l}\text { *Parasit- } \\
\text { ized GW } \\
\text { nymphs / } \\
\text { plant }\end{array}$ & $\begin{array}{l}\text { **Plant } \\
\text { damage } \\
\text { rating }\end{array}$ & $\begin{array}{c}{ }^{*} \mathrm{GW} \text { adults } \\
\text { /plant }\end{array}$ & $\begin{array}{c}{ }^{*} \mathrm{GW} \\
\text { nymphs/plant }\end{array}$ & $\begin{array}{l}\text { *Parasitized } \\
\text { GW nymphs / } \\
\text { plant }\end{array}$ & $\begin{array}{c}\text { **Plant } \\
\text { damage } \\
\text { rating }\end{array}$ & $\begin{array}{c}\text { *GW adults }_{\text {/plant }} \\
\text { G }\end{array}$ & $\begin{array}{c}{ }^{*} \mathrm{GW} \\
\text { nymphs/plant }\end{array}$ & $\begin{array}{l}\text { *Parasit- } \\
\text { ized GW } \\
\text { nymphs / } \\
\text { plant }\end{array}$ & $\begin{array}{l}{ }^{* *} \text { Plant } \\
\text { damage } \\
\text { Rating }\end{array}$ \\
\hline Week 1 & 8-10 leaves & $4.60 \pm 2.33 \mathrm{a}$ & $1.80 \pm 0.20 \mathrm{c}$ & $0.00 \mathrm{~b}$ & $1.00 \mathrm{a}$ & $3.60 \pm 2.33 \mathrm{~b}$ & $2.80 \pm 0.99 \mathrm{c}$ & 0.00 & $1.00 \mathrm{a}$ & $4.20 \pm 1.50 \mathrm{a}$ & $6.00 \pm 3.44 a$ & 0.00 & $1.00 \mathrm{a}$ & $2.40 \pm 1.00 \mathrm{~b}$ & $4.00 \pm 1.45 b$ & 0.00 & $1.00 \mathrm{a}$ \\
\hline Week 2 & 10-12 leaves & $6.00 \pm 3.43 a$ & $1.00 \pm 0.20 \mathrm{~d}$ & $0.00 \mathrm{~b}$ & $1.00 \mathrm{a}$ & $4.80 \pm 1.50 \mathrm{~b}$ & $3.80 \pm 1.78 \mathrm{c}$ & 0.00 & $1.00 \mathrm{a}$ & $5.40 \pm 3.22 \mathrm{~b}$ & $10.80 \pm 5.00 \mathrm{a}$ & 0.00 & $1.00 \mathrm{a}$ & $4.80 \pm 2.01 \mathrm{~b}$ & $6.00 \pm 2.67 \mathrm{~b}$ & 0.00 & $1.00 \mathrm{a}$ \\
\hline Week 3 & 10-12 leaves & $4.80 \pm 1.56 \mathrm{~b}$ & $2.60 \pm 1.10 \mathrm{~d}$ & $0.00 \mathrm{~b}$ & $1.00 \mathrm{a}$ & $4.00 \pm 1.00 \mathrm{~b}$ & $5.60 \pm 2.33 \mathrm{~b}$ & 0.00 & $1.00 \mathrm{a}$ & $6.00 \pm 3.87 \mathrm{a}$ & $9.20 \pm 5.00 \mathrm{a}$ & 0.00 & $1.00 \mathrm{a}$ & $5.00 \pm 2.66 \mathrm{~b}$ & $7.80 \pm 3.11 \mathrm{a}$ & 0.00 & $1.00 \mathrm{a}$ \\
\hline Week 4 & 4-6 laterals & $5.00 \pm 2.00 \mathrm{c}$ & $1.60 \pm 0.89 \mathrm{c}$ & $0.00 \mathrm{~b}$ & $1.00 \mathrm{a}$ & $6.00 \pm 2.88 \mathrm{~b}$ & $3.80 \pm 1.98 \mathrm{c}$ & 0.00 & $1.00 \mathrm{a}$ & $9.00 \pm 4.55 \mathrm{a}$ & $13.20 \pm 6.08 \mathrm{~b}$ & 0.00 & $1.00 \mathrm{a}$ & $6.80 \pm 2.11 \mathrm{~b}$ & $18.60 \pm 6.54 a$ & 0.00 & $3.00 \mathrm{a}$ \\
\hline Week 5 & 6-10 laterals & $2.20 \pm 1.78 \mathrm{c}$ & $2.00 \pm 1.11 \mathrm{c}$ & $4.00 \pm 2.33 \mathrm{a}$ & $1.00 \mathrm{c}$ & $4.00 \pm 1.11 \mathrm{c}$ & $4.00 \pm 2.02 \mathrm{c}$ & $0.00 \mathrm{~b}$ & $1.00 \mathrm{c}$ & $11.60 \pm 4.00 \mathrm{~b}$ & $13.60 \pm 5.99 \mathrm{~b}$ & $4.00 \pm 2.33 \mathrm{a}$ & $2.00 \mathrm{~b}$ & $15.00 \pm 4.67 \mathrm{a}$ & $20.00 \pm 5.11 \mathrm{a}$ & $0.00 \mathrm{~b}$ & $4.00 \mathrm{a}$ \\
\hline Week 6 & $\begin{array}{c}\text { 8-10 laterals, } \\
\text { 16-18 } \\
\text { secondary } \\
\text { laterals }\end{array}$ & $2.20 \pm 0.56 \mathrm{~b}$ & $1.20 \pm 1.00 \mathrm{c}$ & $2.20 \pm 1.23 \mathrm{~b}$ & $1.00 \mathrm{c}$ & $3.80 \pm 2.22 \mathrm{~b}$ & $3.20 \pm 1.54 \mathrm{c}$ & $0.00 \mathrm{c}$ & $1.00 \mathrm{c}$ & $13.00 \pm 5.65 \mathrm{a}$ & $22.00 \pm 5.00 \mathrm{~b}$ & $9.20 \pm 3.33 \mathrm{a}$ & $2.00 \mathrm{~b}$ & $15.00 \pm 5.90 \mathrm{a}$ & $40.60 \pm 8.99 \mathrm{a}$ & $0.00 \mathrm{c}$ & $6.00 \mathrm{a}$ \\
\hline Week 7 & $\begin{array}{l}\text { Flower bud } \\
\text { development }\end{array}$ & $3.00 \pm 1.23 \mathrm{c}$ & $1.00 \pm 0.23 \mathrm{~d}$ & $3.80 \pm 2.44 \mathrm{~b}$ & $1.00 \mathrm{c}$ & $4.00 \pm 2.00 \mathrm{c}$ & $5.00 \pm 3.66 \mathrm{c}$ & $0.00 \mathrm{c}$ & $1.00 \mathrm{c}$ & $15.20 \pm 4.78 b$ & $20.20 \pm 4.98 \mathrm{~b}$ & $11.80 \pm 4.90 \mathrm{a}$ & $2.00 \mathrm{~b}$ & $22.00 \pm 5.33 \mathrm{a}$ & $44.00 \pm 10.22 \mathrm{a}$ & $0.00 \mathrm{c}$ & $6.00 \mathrm{a}$ \\
\hline Week 8 & Flowering & $2.40 \pm 1.00 \mathrm{c}$ & $2.20 \pm 1.22 \mathrm{c}$ & $3.00 \pm 2.56 \mathrm{~b}$ & $1.00 \mathrm{c}$ & $4.60 \pm 3.13 \mathrm{c}$ & $4.40 \pm 2.88 \mathrm{c}$ & $0.00 \mathrm{c}$ & $1.00 \mathrm{c}$ & $13.80 \pm 4.00 \mathrm{~b}$ & $16.60 \pm 8.80 \pm \mathrm{b}$ & $10.60 \pm 3.77 \mathrm{a}$ & $2.00 \mathrm{~b}$ & $20.00 \pm 7.54 \mathrm{a}$ & $48.00 \pm 13.33 \mathrm{a}$ & $0.00 \mathrm{c}$ & $6.00 \mathrm{a}$ \\
\hline Week 9 & Flowering & $2.60 \pm 1.23 \mathrm{c}$ & $1.80 \pm 1.00 \mathrm{c}$ & $3.00 \pm 2.00 \mathrm{~b}$ & $1.00 \mathrm{c}$ & $5.60 \pm 3.05 \mathrm{c}$ & $3.60 \pm 1.67 \mathrm{c}$ & $0.00 \mathrm{c}$ & $1.00 \mathrm{c}$ & $15.00 \pm 5.55 \mathrm{~b}$ & $17.80 \pm 4.00 \mathrm{~b}$ & $13.00 \pm 4.44 \mathrm{a}$ & $2.00 \mathrm{~b}$ & $28.00 \pm 4.44 a$ & $40.00 \pm 10.34 \mathrm{a}$ & $0.00 \mathrm{c}$ & $6.00 \mathrm{a}$ \\
\hline Week 10 & Flowering & $3.00 \pm 1.54 \mathrm{c}$ & $1.20 \pm 0.76 \mathrm{~d}$ & $4.00 \pm 1.99 \mathrm{~b}$ & $1.00 \mathrm{c}$ & $6.20 \pm 3.44 \mathrm{c}$ & $4.20 \pm 2.33 c$ & $0.00 \mathrm{c}$ & $2.00 \mathrm{~b}$ & $14.40 \pm 5.67 \mathrm{~b}$ & $10.00 \pm 3.99 \mathrm{~b}$ & $8.60 \pm 4.00 \mathrm{a}$ & $2.00 \mathrm{~b}$ & $26.20 \pm 6.76 \mathrm{a}$ & $53.00 \pm 15.01 \mathrm{a}$ & $0.00 \mathrm{c}$ & $7.00 \mathrm{a}$ \\
\hline Week 11 & Flowering & $3.60 \pm 0.98 \mathrm{c}$ & $1.80 \pm 0.86 \mathrm{~d}$ & $4.00 \pm 3.44 \mathrm{~b}$ & $1.00 \mathrm{c}$ & $7.60 \pm 2.90 \mathrm{c}$ & $5.60 \pm 3.44 \mathrm{c}$ & $0.00 \mathrm{c}$ & $2.00 \mathrm{~b}$ & $15.00 \pm 5.66 \mathrm{~b}$ & $14.60 \pm 5.00 \mathrm{~b}$ & $8.40 \pm 2.00 \mathrm{a}$ & $2.00 \mathrm{~b}$ & $29.00 \pm 5.67 \mathrm{a}$ & $65.00 \pm 15.32 \pm \mathrm{a}$ & $0.00 \mathrm{c}$ & $7.00 \mathrm{a}$ \\
\hline Week 12 & Flowering & $4.80 \pm 1.34 \mathrm{c}$ & $2.20 \pm 0,88 \mathrm{c}$ & $2.00 \pm 0.99 \mathrm{~b}$ & $1.00 \mathrm{c}$ & $4.20 \pm 3.44 \mathrm{c}$ & $4.60 \pm 2.66 \mathrm{c}$ & $0.00 \mathrm{c}$ & $2.00 \mathrm{~b}$ & $13.20 \pm 4.87 \mathrm{~b}$ & $21.20 \pm 7.77 \mathrm{~b}$ & $10.60 \pm 4.44 \mathrm{a}$ & $2.00 \mathrm{~b}$ & $30.00 \pm 8.22 \mathrm{a}$ & $60.00 \pm 7.54 \mathrm{a}$ & $0.00 \mathrm{c}$ & $7.00 \mathrm{a}$ \\
\hline Week 13 & Flowering & $1.60 \pm 0.76 c$ & $1.00 \pm 0.49 \mathrm{~d}$ & $2.60 \pm 1.22 \mathrm{~b}$ & $1.00 \mathrm{c}$ & $5.00 \pm 2.30 \mathrm{c}$ & $3.00 \pm 1.87 \mathrm{c}$ & $0.00 \mathrm{c}$ & $2.00 \mathrm{~b}$ & $14.00 \pm 4.33 b$ & $16.60 \pm 5.60 \mathrm{~b}$ & $13.20 \pm 5.65 \mathrm{a}$ & $2.00 \mathrm{~b}$ & $27.00 \pm 5.66 \mathrm{a}$ & $62.00 \pm 13.45 \mathrm{a}$ & $0.00 \mathrm{c}$ & $8.00 \mathrm{a}$ \\
\hline Week 14 & Flowering & $2.00 \pm 1.03 \mathrm{c}$ & $1.00 \pm 0.34 \mathrm{~d}$ & $3.20 \pm 0.80 \mathrm{~b}$ & $1.00 \mathrm{~d}$ & $4.00 \pm 1.99 \mathrm{c}$ & $4.80 \pm 2.88 \mathrm{c}$ & $0.00 \mathrm{c}$ & $2.00 \mathrm{c}$ & $17.00 \pm 4.00 \mathrm{~b}$ & $14.20 \pm 3.56 \mathrm{~b}$ & $11.00 \pm 3.44 \mathrm{a}$ & $3.00 \mathrm{~b}$ & $28.00 \pm \mathrm{a} 6.77$ & $96.40 \pm 10.11 \mathrm{a}$ & $0.00 \mathrm{c}$ & $9.00 \mathrm{a}$ \\
\hline Week 15 & Flowering & $4.00 \pm 2.22 \mathrm{c}$ & $1.00 \pm 0.25 \mathrm{~d}$ & $4.00 \pm 2.11 \mathrm{~b}$ & $1.00 \mathrm{~d}$ & $4.00 \pm 2.76 \mathrm{c}$ & $4.60 \pm 3.40 \mathrm{c}$ & $0.00 \mathrm{c}$ & $2.00 \mathrm{c}$ & $15.00 \pm 5.55 \mathrm{~b}$ & $16.00 \pm 4.00 \mathrm{~b}$ & $12.20 \pm 5.43 \mathrm{a}$ & $3.00 \mathrm{~b}$ & $30.00 \pm 7.10 \mathrm{a}$ & $90.00 \pm 13.43 \mathrm{a}$ & $0.00 \mathrm{c}$ & $9.00 \mathrm{a}$ \\
\hline Week 16 & Flowering & $2.00 \pm 1.01 \mathrm{c}$ & $2.00 \pm 0.88 \mathrm{c}$ & $4.40 \pm 3.45 \mathrm{~b}$ & $1.00 \mathrm{~d}$ & $3.60 \pm 2.02 \mathrm{c}$ & $5.80 \pm 3.22 \mathrm{c}$ & $0.00 \mathrm{c}$ & $2.00 \mathrm{c}$ & $13.60 \pm 5.44 \mathrm{~b}$ & $12.00 \pm 5.05 \mathrm{~b}$ & $12.80 \pm 4.00 \mathrm{a}$ & $3.00 \mathrm{~b}$ & $26.00 \pm 8.89 \mathrm{a}$ & $82.20 \pm 12.22 \mathrm{a}$ & $0.00 \mathrm{c}$ & $9.00 \mathrm{a}$ \\
\hline Week 17 & Flowering & $3.20 \pm 1.89 \mathrm{c}$ & $2.60 \pm 1.00 \mathrm{c}$ & $3.00 \pm 1.99 \mathrm{~b}$ & $1.00 \mathrm{~d}$ & $3.60 \pm \mathrm{c}$ & $4.00 \pm \mathrm{c}$ & $0.00 \mathrm{c}$ & $2.00 \mathrm{c}$ & $15.00 \pm \mathrm{b}$ & $23.60 \pm b$ & $8.40 \pm \mathrm{a}$ & $3.00 \mathrm{~b}$ & $31.00 \pm \mathrm{a}$ & $94.00 \pm \mathrm{a}$ & $0.00 \mathrm{c}$ & $9.00 \mathrm{a}$ \\
\hline Week 18 & Flowering & $2.60 \pm 1.76 \mathrm{~d}$ & $3.80 \pm 1.34 \mathrm{c}$ & $4.00 \pm 2.43 \mathrm{~b}$ & $1.00 \mathrm{~d}$ & $5.00 \pm \mathrm{c}$ & $5.20 \pm \mathrm{c}$ & $0.00 \mathrm{c}$ & $2.00 \mathrm{c}$ & $16.00 \pm b$ & $12.20 \pm \mathrm{b}$ & $12.00 \pm \mathrm{a}$ & $3.00 \mathrm{~b}$ & $33.00 \pm \mathrm{a}$ & $106.00 \pm \mathrm{a}$ & $0.00 \mathrm{c}$ & $9.00 \mathrm{a}$ \\
\hline Week 19 & Flowering & $2.00 \pm 0.97 \mathrm{c}$ & $2.80 \pm 1.00 \mathrm{c}$ & $4.60 \pm 2.00 \mathrm{~b}$ & $1.00 \mathrm{~d}$ & $4.20 \pm c$ & $3.60 \pm \mathrm{c}$ & $0.00 \mathrm{c}$ & $2.00 \mathrm{c}$ & $12.80 \pm \mathrm{b}$ & $15.00 \pm \mathrm{b}$ & $0 \pm \mathrm{a}$ & $3.00 \mathrm{~b}$ & $24.00 \pm \mathrm{a}$ & $125.00 \pm \mathrm{a}$ & $0.00 \mathrm{c}$ & $9.00 \mathrm{a}$ \\
\hline Week 20 & Flowering & $2.80 \pm 1.00 \mathrm{c}$ & $1.00 \pm 0.50 \mathrm{~d}$ & $3.60 \pm 2.99 \mathrm{~b}$ & $1.00 \mathrm{~d}$ & $4.80 \pm \mathrm{c}$ & $4.00 \pm \mathrm{c}$ & $0.00 \mathrm{c}$ & $2.00 \mathrm{c}$ & $9.60 \pm \mathrm{b}$ & $12.00 \pm b$ & $10.00 \pm \mathrm{a}$ & $3.00 \mathrm{~b}$ & $20.00 \pm \mathrm{a}$ & $120.80 \pm \mathrm{a}$ & $0.00 \mathrm{c}$ & $9.00 \mathrm{a}$ \\
\hline
\end{tabular}

${ }^{1}$ E. formosa@ 3 per m² weekly+Amblyseius cucumeris@ 50 per m² biweekly; ${ }^{2}$ E. formosa@ 3 per m ${ }^{2}$ weekly; ${ }^{3}$ Amblyseius cucumeris @ 50 per $m^{2}$ biweekly; ${ }^{4}$ Control: Untreated plants

*Means represent 2-year averages of 25 plants per treatment (4 treatments, 5 replications, 5 plants per replication, 100 plants in total)

***Plant damage rating: $1-9$ scale (\% leaf area affected): $1=0-5,2=6-10,3=11-20,4=21-30,5=31-40,6=41-50,7=51-60,8=61-70,9=>70$

Number followed by the same letter are not significantly different in LSD at $\mathrm{P}=0.05$ 
One hundred plants were randomly selected and grouped into 4 treatments consisting of 25 plants each. The treatment groups were: 1. E. formosa + Amblyseius cucumeris, 2. Amblyseius cucumeris, 3. E. formosa, and 4. untreated control. The layout and setup of the experiment were the same as experiment 1. E. formosa was received in paper cards containing pupae within a parasitized host; cards were placed in plant pots by staking into the growing medium. The rate of application used for E. formosa was 3 pupae per $\mathrm{m}^{2}$ at weekly intervals as recommended by the manufacturer. Amblyseius cucumeris was applied at the rate of 50 mites per $\mathrm{m}^{2}$ (instead of the manufacturer's recommended rate of 100 mites per $\mathrm{m}^{2}$ ) at biweekly intervals. Plants were placed in close contact with their foliage touching to promote mite dispersal. GW adults, nymphs, and parasitized nymphs on all the above-ground plant parts were counted at the weekly interval, and means were calculated. The initial number of GW adults and nymphs at the start of monitoring (week 1) ranged from 2.40 to 4.60 , and 1.80 to 6.00 per plant respectively. The damage symptoms on plants were recorded using a rating scale of 1 to 9 , the same as used in experiment 1 .

Experiment 4: Efficacy of Aphidius colemani and Aphidius ervi for the control of aphids (Green peach aphids -M. persicae, Foxglove aphids- A. solani) on pansy (Viola $x$ wittrockiana 'Matrix ${ }^{\circledR}$ Citrus Mixture').

The pansy (Viola $x$ wittrockiana 'Matrix ${ }^{\circledR}$ Citrus Mixture') crop was chosen to carry out this experiment due to its susceptibility to an aphid attack. The transplanted plants grew vegetatively until week 7 and flowered thereafter (Table 4).

Table 4. Effect of Aphidius colemani and Aphidius ervi on aphids (Green peach aphids - M.persicae, Foxglove aphids- A.solani) in pansy

\begin{tabular}{|c|c|c|c|c|c|c|c|}
\hline & \multirow{2}{*}{$\begin{array}{c}\text { Plant } \\
\text { Growth } \\
\text { Stage }\end{array}$} & \multicolumn{3}{|c|}{${ }^{1}$ Aphidius colemani + Aphidius ervi } & \multicolumn{3}{|c|}{${ }^{2}$ Control } \\
\hline & & $\begin{array}{l}* * \text { Aphids per } \\
\text { plant }\end{array}$ & $\begin{array}{l}* * \text { Parasitized } \\
\text { aphids per plant }\end{array}$ & $\begin{array}{c}* * * \text { Plant } \\
\text { damage rating }\end{array}$ & $\begin{array}{l}* * \text { Aphids per } \\
\text { plant }\end{array}$ & $\begin{array}{c}* * \text { Parasitized } \\
\text { aphids per } \\
\text { plant }\end{array}$ & $\begin{array}{c}* * * \text { Plant } \\
\text { damage } \\
\text { rating }\end{array}$ \\
\hline Week 1 & 5-8 leaves & $15.40 \pm 5.00 \mathrm{a}$ & 0.00 & $1.00 \mathrm{a}$ & $11.00 \pm 4.55 b$ & 0.00 & $1.00 \mathrm{a}$ \\
\hline *Week 2 & 5-8 leaves & $16.00 \pm 5.78 \mathrm{a}$ & $0.60 \pm 0.40 \mathrm{a}$ & $1.00 \mathrm{a}$ & $12.50 \pm 5.00 \mathrm{~b}$ & $0.00 \mathrm{~b}$ & $1.00 \mathrm{a}$ \\
\hline *Week 3 & 8-10 leaves & $18.20 \pm 7.65 \mathrm{a}$ & $0.80 \pm 1.00 \pm \mathrm{a}$ & $1.00 \mathrm{a}$ & $20.40 \pm 4.44 a$ & $0.00 \mathrm{~b}$ & $1.00 \mathrm{a}$ \\
\hline *Week 4 & 8 -10 leaves & $22.00 \pm 5.00 \mathrm{~b}$ & $1.50 \pm 1.01 \mathrm{a}$ & $1.00 \mathrm{a}$ & $26.00 \pm 6.05 \mathrm{a}$ & $0.00 \mathrm{~b}$ & $1.00 \mathrm{a}$ \\
\hline *Week 5 & 10-12 leaves & $25.60 \pm 7.89 \mathrm{a}$ & $3.20 \pm 0.33 \mathrm{a}$ & $1.00 \mathrm{a}$ & $22.00 \pm 4.44 \mathrm{a}$ & $0.00 \mathrm{~b}$ & $1.00 \mathrm{a}$ \\
\hline Week 6 & 12-15 leaves & $20.20 \pm 10.00 b$ & $15.60 \pm 4.32 \mathrm{a}$ & $1.00 \mathrm{~b}$ & $30.80 \pm 6.18 \mathrm{a}$ & $0.00 \mathrm{~b}$ & $3.00 \mathrm{a}$ \\
\hline Week 7 & 18-20 leaves & $18.40 \pm 5.89 b$ & $17.00 \pm 7.87 \mathrm{a}$ & $1.00 \mathrm{~b}$ & $42.00 \pm 10.09 a$ & $0.00 \mathrm{~b}$ & $3.00 \mathrm{a}$ \\
\hline Week 8 & $\begin{array}{c}\text { 20-25 leaves, } \\
\text { flowering }\end{array}$ & $16.60 \pm 7.77 b$ & 0.00 & $1.00 \mathrm{~b}$ & $56.60 \pm 12.20 \mathrm{a}$ & 0.00 & $5.00 \mathrm{a}$ \\
\hline Week 9 & Flowering & $6.60 \pm 2.88 \mathrm{~b}$ & $26.00 \pm 6.34 \mathrm{a}$ & $1.00 \mathrm{~b}$ & $59.60 \pm 9.89 \mathrm{a}$ & $0.00 \mathrm{~b}$ & $5.00 \mathrm{a}$ \\
\hline Week 10 & Flowering & $5.50 \pm 2.00 \mathrm{~b}$ & $38.80 \pm 10.00 \mathrm{a}$ & $1.00 \mathrm{~b}$ & $60.20 \pm 13.44 \mathrm{a}$ & $0.00 \mathrm{~b}$ & $5.00 \mathrm{a}$ \\
\hline Week 11 & Flowering & $5.00 \pm 3.11 \mathrm{~b}$ & $52.20 \pm 12.00 \mathrm{a}$ & $1.00 \mathrm{~b}$ & $88.80 \pm 15.67 \mathrm{a}$ & $0.00 \mathrm{~b}$ & $6.00 \mathrm{a}$ \\
\hline Week 12 & Flowering & $3.20 \pm 0.99 b$ & $48.80 \pm 8.88 \mathrm{a}$ & $1.00 \mathrm{~b}$ & $102.00 \pm 26.76 \mathrm{a}$ & $0.00 \mathrm{~b}$ & $7.00 \mathrm{a}$ \\
\hline Week 13 & Flowering & $4.40 \pm 1.67 b$ & $60.00 \pm 11.12 \mathrm{a}$ & $1.00 \mathrm{~b}$ & $120.40 \pm 23.09 a$ & $0.00 \mathrm{~b}$ & $7.00 \mathrm{a}$ \\
\hline Week 14 & Flowering & $2.00 \pm 1.00 \mathrm{~b}$ & $62.00 \pm 12.00 \mathrm{a}$ & $1.00 \mathrm{~b}$ & $122.40 \pm 17.89 a$ & $0.00 \mathrm{~b}$ & $7.00 \mathrm{a}$ \\
\hline Week 15 & Flowering & $1.60 \pm 3.00 \mathrm{~b}$ & $68.40 \pm 9.98 \mathrm{a}$ & $1.00 \mathrm{~b}$ & $158.00 \pm 32.34 \mathrm{a}$ & $0.00 \mathrm{~b}$ & $8.00 \mathrm{a}$ \\
\hline Week 16 & Flowering & $1.60 \pm 1.42 b$ & $68.20 \pm 10.89 a$ & $1.00 \mathrm{~b}$ & $162.00 \pm 22.34 \mathrm{a}$ & $0.00 \mathrm{~b}$ & $8.00 \mathrm{a}$ \\
\hline Week 17 & Flowering & $1.00 \pm 0.33 b$ & $72.20 \pm 18.90 \mathrm{a}$ & $1.00 \mathrm{~b}$ & $179.80 \pm 39.89 a$ & $0.00 \mathrm{~b}$ & $9.00 \mathrm{a}$ \\
\hline Week 18 & Flowering & $1.80 \pm 1.00 \mathrm{~b}$ & $70.00 \pm 11.11 \mathrm{a}$ & $1.00 \mathrm{~b}$ & $169.80 \pm 35.56 \mathrm{a}$ & $0.00 \mathrm{~b}$ & $9.00 \mathrm{a}$ \\
\hline Week 19 & Flowering & $1.00 \pm 0.20 \mathrm{~b}$ & $62.40 \pm 8.88 \mathrm{a}$ & $1.00 \mathrm{~b}$ & $180.80 \pm 25.67 \mathrm{a}$ & $0.00 \mathrm{~b}$ & $9.00 \mathrm{a}$ \\
\hline Week 20 & Flowering & $1.00 \pm 0.33 b$ & $72.00 \pm 13.43 a$ & $1.00 \mathrm{~b}$ & $188.80 \pm 32.22 \mathrm{a}$ & $0.00 \mathrm{~b}$ & $9.00 \mathrm{a}$ \\
\hline
\end{tabular}

${ }^{1}$ Aphidius colemani + A.ervi (mix)@3 per m² during *weeks 2,3,4,5

${ }^{2}$ Control=Untreated plants

**Means represent 2-year averages of 25 plants per treatment ( 2 treatments, 5 replications, 5 plants per replication, 50 plants in total)

$* * *$ Plant damage rating: $1-9$ scale ( $\%$ leaf area affected): $1=0-5,2=6-10,3=11-20,4=21-30,5=31-40,6=41-50,7=51-60,8=61-70,9=>70$

Number followed by the same letter are not significantly different in LSD at $\mathrm{P}=0.05$ 
Fifty randomly selected plants were divided into 2 treatment groups of 25 plants each. The treatment groups were: 1) Aphidius colemani + Aphidius ervi, 2) untreated control. The setup and layout of the experiment were the same as experiment 1. A mixture of Aphidius colemani and Aphidius ervi wasps were received as pupae within the aphid golden mummies, ready to emerge as adults. We used the recommended rate of application of 3 pupae per $\mathrm{m}^{2}$. Although the manufacturers recommended applying Aphidius mix at weekly intervals, we only applied the mix during weeks 2, 3, 4, and 5. Every aphid and parasitized nymph on all above-ground plant parts was counted every week and means were calculated. The initial number of aphids during week 1 was 11.00 and 15.40 per plant in the treated and control treatments respectively. The damage symptoms on plants were recorded using a rating scale of 1 to 9 , the same as used in experiment 1.

\section{Results and Discussion}

Experiment 1: Efficacy of $P$. persimilis for the control of two-spotted spider mites (TSSM), T. urticae, on calibrachoa (Calibrachoa $x$ hybrida 'Conga' ${ }^{\mathrm{TM}}$ Deep Yellow').

$P$. persimilis controlled TSSM in pansies successfully, and we were able to achieve positive results with only four applications of $P$. persimilis in 20 weeks. The TSSM remained suppressed and numbers remained low for most of the study period. After the first application of $P$. persimilis in week $6,21.43 \%$ reduction in TSSM was observed in week 8 , which further plunged to $69.05 \%$ in week 9. Significantly higher numbers of TSSM (144.40 per plant in week 20) were recorded in untreated plants in comparison to treated plants (Table 1). Untreated plants had a high level of damage symptoms from week 8 onwards. Graying or yellowing of the leaves was observed from week 5 onwards, and necrotic spots developed on leaves by week 8 . Flowers showed browning and withering of the petals. All plant parts were covered by silky webbing material secreted by mites. Towards the end of the trial, plants displayed a stippled-bleached effect with most of the leaves turning yellow, gray, or bronze, followed by defoliation. No visible damage on $P$. persimilis treated plants were observed through most of the growing cycle of the crop.

The reasons for high efficacy for $P$. persimilis may have been the high relative humidity ( $\mathrm{RH}-50 \%-80 \%$ range) and high plant density in our greenhouse. $P$. persimilis eggs are highly sensitive to constant low humidity, with only $20 \%$ of the eggs hatch at constant low relative humidity conditions. Under variable humidity conditions (as in our case), eggs can compensate for water loss during periods of higher humidity (Hesran et al., 2019). Close plant contact also improves the dispersal ability of $P$. persimilis. (Pundt, 2014). The temperature in our greenhouse fluctuated throughout the trial period and was higher than $35^{\circ} \mathrm{C}$ on hot and sunny days in June and July which resulted in a surge in TSSM during summer. Abo-Elmaged et al. (2020) reported that temperature, relative humidity, and plant age play an important role in the TSSM infestation than any other abiotic and biotic factors. Although the development time for $P$. persimilis is shorter than for spider mites $(5$ days at $30{ }^{\circ} \mathrm{C}, 9$ days at $20^{\circ} \mathrm{C}$, and 25 days at $15^{\circ} \mathrm{C}$ ), at high temperatures above $30^{\circ} \mathrm{C}$ TSSM develops at a much faster rate and $P$. persimilis may be unable to match its reproductive abilities (Pundt, 2014).

Experiment 2: Efficacy of Amblyseius cucumeris and S. feltiae for the control of western flower thrips (WFT), F. occidentalis, on sweet potato vine (Ipomoea batatas 'Marguerite').

The predator/parasitoid complex, Amblyseius cucumeris and $S$. feltiae resulted in an overall reduction of the WFT population by $95.04 \%$ on plants ( 1.40 mean number of WFT per plant) and $99.00 \%$ on sticky card (1.30 mean number of WFT per sticky card) from their starting population in a 20 -week trial period. We observed the time taken for the first significant population reduction of WFT was 4 weeks for the Amblyseius cucumeris $+S$. feltiae treatment (Table 2). After application to the soil, it probably took time for the nematodes to reach the depth at which WFT pupated, and during this period, some thrips reached the adult stage, escaping from the effect of $S$. feltiae. Once in contact, $S$. feltiae can enter its host in multiple ways through mouth, anus, and spiracles (Chergui et al., 2019). S. feltiae infests WFT's at pre-pupae and pupal stage in the soil (Buitenhuis and Shipp, 2005), while Amblyseius cucumeris feeds on all above-ground life stages of WFT, except pupae (in soil) and eggs (inside leaf tissue) (Sarwar, 2016). This explains why our results for WFT management were better when we used Amblyseius cucumeris and S. feltiae together, as the combination provided better coverage for all stages of WTF.

The positive effect of the first application of Amblyseius cucumeris (alone) was observed during week 6 where we noticed a sharp decrease in WFT on plants by $65.38 \%$ and on sticky cards by $82.01 \%$ in comparison to WTF numbers during week 1. During the last week of trial, we recorded an average of 3.20 WFT per plant $(84.62 \%$ lower than the starting population) and 13.00 WFT per sticky card (88.54\% lower than the starting population) with this treatment (Table 2). The occasional decline in the performance of Amblyseius cucumeris could be due to suboptimal temperature and humidity in the greenhouse on which the success of predation depends, and also due to the mode of its release in the crop. The environmental stresses can adversely affect the performance of slowrelease sachets of predatory mites (Shimoda et al., 2017). The emergence patterns of Amblyseius cucumeris from the sachets in the greenhouse could be highly variable even under ideal climatic conditions (Buitenhuis and Brommit, 2014). Mikawa et al. (2020) installed the predator release system in the greenhouse found approximately 1 month is necessary for the distribution of the released $N$. californicus on the leaves. Another reason for Amblyseius cucumeris to be less effective could be the low density of prey (Delisle et al., 2015). In our experiments, the damage symptoms on the plants were significantly higher on 
control plants in comparison to treatment using Amblyseius cucumeris alone or in combination with $S$. feltiae. Severe damage occurred in control plants throughout the 20 weeks resulting in unmarketable plants. Leaves of untreated plants developed brownish silvery speckling and white patches. Growing points of some plants became distorted (Table 2).

We did not use $S$. feltiae alone as a biocontrol agent in our experiment. Since the level of WTF control is affected by the concentration of nematodes applied in the medium (Saito and Brownbridge, 2016), the cost of application of $S$. feltiae alone at the rate appropriate to regulate WFT populations with the desired mortality rates of greater than $80 \%$ was too expensive (Arthurs and Heinz, 2006).

Experiment 3: Efficacy of E. formosa and Amblyseius cucumeris for the control of greenhouse whitefly (GW), T. vaporariorum on calibrachoa (Calibrachoa $x$ hybrida 'Chameleon ${ }^{\circledR}$ ')

A reasonable level of parasitism was seen with the use of E. Formosa in our trial. However, using this parasitoid alone was ineffective in bringing the GW densities below the threshold levels of 3-4 nymphs per plant. Research shows that weather conditions (humidity, wind speed, and temperature) affect the foraging behaviour of parasitoids (Vosteen et al. 2020). Both flying ability (Van Roermund and Van Lenteren, 1995) and walking activity (Netting and Hunter, 2000) of E. formosa are affected at temperatures below $18{ }^{\circ} \mathrm{C}$, lowering the parasitoid's ability to find and parasitize GW. The temperature during our experiment dropped below $18{ }^{\circ} \mathrm{C}$ for a few days in March which explains why we did not record any signs of parasitism until week 5 in our experiments. The past research explained the influence of light intensity and daylength on feeding and oviposition activity of aphelinid parasitoids, E. formosa, and Eretmocerus eremicus. Both biocontrol agents parasitized significantly more whiteflies under the simulated summer (i.e., high light intensity [82.0-83.6 W $\mathrm{m}^{-2}$, long day length [L 16:D $8 \mathrm{~h}$ ], $24{ }^{\circ} \mathrm{C}$ ) treatment than the winter (i.e., low light intensity [10.8- $11.1 \mathrm{~W} \mathrm{~m}^{-2}$ ], short day length [L 8:D $16 \mathrm{~h}$ ], 20 ${ }^{\circ} \mathrm{C}$ ) treatment (Zilahi-Balogh et al., 2006). The long daylength of [L 16:D $8 \mathrm{~h}$ ] is generally observed in June and July months in Manitoba, whereas most of the production of bedding plants is done from March to May. The inability to achieve a higher parasitism rate in our study might also be due to the stronger influence of fluctuating temperatures than that of day length.

The GW adult and nymph densities recorded with the Amblyseius cucumeris treatment were significantly lower than those in the E. formosa treated, and also in untreated control plants. There were only minor weekly increases of GW nymphs per plant $(\mathrm{P}>0.05)$; starting population of 2.80 nymphs per plant was the lowest number observed and 5.80 nymphs per plant was the highest observed in week 16 (Table 3). The benefit of using Amblyseius cucumeris in flowering crops is that this predatory mite can develop and reproduce feeding on non-prey food sources such as pollen, which allows populations of the predator to build upon plants before the pests are present and to persist in the crop during periods when prey is scarce or absent (Nemati et al., 2019). High-density close planting of bedding plants also facilitated the dispersal of the Amblyseius cucumeris in our greenhouse resulting in the success of this biocontrol treatment. An additional advantage of using Amblyseius cucumeris in the greenhouses is its effectiveness as a predator for western flower thrips along with whiteflies.

Although Amblyseius cucumeris alone also proved to be an effective predator for GW, however, due to less consistent temperatures and relative humidity in the greenhouse during the growing cycle, it was still unable to bring the GW densities below threshold levels. That is why we included E. formosa alongside Amblyseius cucumeris, as an added layer of protection to parasitize GW nymphs that escaped the predation of Amblyseius cucumeris. The combination treatment of E. formosa and Amblyseius cucumeris proved to be a significantly better treatment for control of GW in the crop than the other three treatments. This treatment brought the GW densities below threshold levels and helped maintain a high quality of plants in our study (Table 3 ).

As far as plant damage is concerned, severe damage symptoms were observed from week 6 onwards in the untreated control plants. Yellowing, malformation, and premature fall of leaves occurred in almost all of the plants. In contrast, no visible symptoms were found on the plants treated with E. formosa + Amblyseius cucumeris treatment. The use of E. formosa alone resulted in having a few plants with slight damage; the quality of plants was not premium, but they were marketable.

Experiment 4: Efficacy of Aphidius colemani and Aphidius ervi for the control of aphids (Green peach aphidsM. persicae, Foxglove aphids- A. solani) on pansy (Viola $x$ wittrockiana 'Matrix ${ }^{\circledR}$ Citrus Mixture')

We started our trial with a high initial population of aphids in the greenhouse. We carried out the first application of the Aphidius mix during the second week of the trial period and continued the application of this mix for the next 3 weeks. Aphid densities displayed linear growth from week 1 to 5 . This could be attributed to the defense strategies adopted by older and larger aphids to protect themselves from parasitoids by kicking, dropping, shaking their body, and running away (Vorburger, 2018). From week 6 to 8 , the aphid density declined slowly, but from week 9 onwards it plummeted sharply. A steady increase of parasitized aphids per plant was noticed through to the 20 -week trial period, resulting in a mean of 72.00 parasitized aphids per plant. A linear increase in the number of aphids per plant was observed from week 1 (11.00 per plant) to week 20 (188.80 aphids per plant) resulting in an escalation of $94.17 \%$ in untreated plants. (Table 4). The manufacturer's recommendation was to apply the Aphidius mix every week. However, in our case, we were able to establish the self-sustaining parasitizing cycle of Aphidius spp. on aphids just with four successive weekly applications. There are many possible reasons for the successful control of aphids by the Aphidius mix in our study. Firstly, Aphidius colemani is very efficient in adapting itself to respond to changing host densities. 
It exhibits a type III functional response on low-density patches (linear increase in parasitism with increasing host density, until a maximum is reached) while exhibiting a type II functional response (decreasing parasitism with increasing host density) at higher aphid densities (Prado et al., 2015). Secondly, adults of Aphidius spp. are extremely strong flyers and females can fly 16 meters within 24 hours after release in search of aphids (Langhof et al., 2005, Jennifer et al., 2018). Thirdly, Aphidius spp. are not impacted by short day length, so it is possible to use them year-round at optimum temperatures. Additionally, adult parasitoids of Aphidius spp. often consume flower nectar for carbohydrates and other nutrients (Goelen et al., 2018); such resource provisioning by plants can benefit parasitoid life-history traits and parasitism. Lastly, Aphidius spp. is adaptive and tolerant to abiotic factors like low relative humidity. There were no differences in foraging abilities (residence times, time allocation, or oviposition success) for $A$. rosae when exposed to low humidity in the field of $40 \%$ critical relative humidity (Fink and Volkl, 1995).

\section{Conclusions}

Overall, in our study, the biocontrol agents were as effective as chemical pesticides for managing a variety of pests in a commercial greenhouse setting. The growers informed us that they used $70 \%$ less chemical pesticides than in previous years without observing any deterioration in the quality of plants. So far, no information is documented about how climatic conditions like that of Canadian prairies affect the greenhouse environment, and how the latter influence pest management strategies for greenhouse pests. Therefore, until now, the only option for our growers was to rely on recommendations on biocontrol use from greenhouses located in other parts of Canada or Europe where climatic conditions are not similar to ours. The results of our study demonstrate that there was no adverse effect of prairie environmental conditions on the efficacy of biocontrol agents. We tried many different combinations of biocontrol agents and conclude that the combinations of tried biocontrol agents worked well together without interfering with one another to reduce efficiency. The information presented in the study could be of great value to floricultural greenhouse growers in Canada and elsewhere in the world where similar climatic conditions prevail. We anticipate that the success of this program will encourage other greenhouses to grow their crops using more sustainable and eco-friendly practices. Furthermore, the present study could create possibilities for growing food in the greenhouses for remote and isolated communities of the north where food insecurity is extremely high.

\section{Author Contribution}

PS: obtaining funds through a research grant, arranging an industry partner to carry out this research in a commercial greenhouse, planning and designing of research experiments, data analysis and interpretation, and writing of the manuscript. TN: data collection, and maintaining a regular communication with industry partner.

\section{Acknowledgments}

The authors thank Mr. Chad Labbe, Owner and CEO of Shelmerdine Garden Center for allowing us to work in the nursery. The funding for the project was provided by the Agriculture and Agri-Food Canada and the Manitoba Ministry of Agriculture through the Canada-Manitoba Growing Forward 2 program, and the National Science and Engineering Research Council of Canada. Trade names or commercial products mentioned in this publication is solely to provide specific information and does not imply recommendation or endorsement by Assiniboine Community College.

\section{References}

ABO-ELMAGED, T.M.; ABDEL-WAHAB M.A.; ABDELRAHMAN M.A.A.; ABD-ALLAH, A.H.A. Activity of the two-spotted spider mite, Tetranychus urticae (Koch) (Acari) infesting cucumber plants in upper Egypt. International Journal Tropical Insect Science, 2020. v.41, p.463-469. ttps://doi.org/10.1007/s42690-020-00232-6

ALIPOUR, Z.; FATHIPOUR, Y.; FARAZMAND, A.; KHANAMANI, M. Resistant rose cultivar affects life table parameters of two-spotted spider mite and its predators Phytoseiulus persimilis and Amblyseius swirskii (Phytoseiidae). Systematic and Applied Acarology, v.24, n.9, p.1620-1630, 2019. https://doi. org/10.11158/saa.24.9.4

ARTHURS, S.; HEINZ, K.M. Evaluation of the nematodes Steinernema feltiae and Thripinema nicklewoodi as biological control agents of western flower thrips Frankliniella occidentalis infesting chrysanthemum. Biocontrol Science and Technology, v.6, n.2, p.141-155, 2006. https://doi. org/10.1080/09583150500258545

BROWNBRIDGE, M. 2017. How to successfully integrate biocontrols into greenhouse floriculture production. Greenhouse Grower. Available at: <https://www. greenhousegrower.com/production/insect-control/howto-successfully-integrate-biocontrols-into-greenhousefloriculture-production/> Accessed on: February 9 ${ }^{\text {th }}, 2021$.

BUITENHUIS, R.; SHIPP, J.L. Efficacy of entomopathogenic nematode Steinernema feltiae (Rhabditida: Steinernematidae) as influenced by Frankliniella occidentalis (Thysanoptera: Thripidae) developmental stage and host plant stage. Journal of Economic Entomology, v.98, n.5, p.1480-1485, 2005. https://doi.org/10.1093/jee/98.5.1480

BUITENHUIS, E.G.; BROMMIT, A. Practical placement improves the performance of slow-release sachets of Neoseiulus cucumeris. Biocontrol Science and Technology, v.24, n.10, p.1153-1166, 2014. https://doi.or $\mathrm{g} / 10.1080 / 09583157.2014 .930726$ 
CHERGUI, S.; BENZEHRA, A.; BOUDJEMAA, K.; BARKOU H.; KARACA, I. Efficacy of Turkish isolate of Steinernema feltiae (Rhabditida: Steinernematidae) in controlling the Mediterranean fruit fly, Ceratitis capitata (Wiedemann) (Diptera: Tephritidae), under laboratory conditions. Egyptian Journal of Biological Pest Control, v.29, p.60, 2019. https://doi.org/10.1186/ s41938-019-0162-4

DELISLE, J.F.; SHIPP, L.; BRODEUR, J. 2015. Apple pollen as a supplemental food source for the control of western flower thrips by two predatory mites, Amblyseius swirskii and Neoseiulus cucumeris (Acari: Phytoseiidae), on potted chrysanthemum. Experimental and Applied Acarology, v.65, p.495-509, 2015. https://doi.org/10.1007/ s10493-014-9863-2

FINK, U.; VOLKL, W. The effect of abiotic factors on foraging and oviposition success of the aphid parasitoid, Aphidius rosae. Oecologia, v.103, p.371-378, 1995. https:// doi.org/10.1007/BF00328627

GOELEN, T.; BAETS, D.; KOS, M.; PAULUSSEN, C.; LENAERTS, M.; REDIERS, H.; WÄCKERS, F.; JACQUEMYN, H.; LIEVENS, B. Gustatory response and longevity in Aphidius parasitoids and their hyperparasitoid Dendrocerus aphidum. Journal of Pest Science, v.91, p.351-360, 2018. https://doi.org/10.1007/ s10340-017-0907-3

GARRIGA, A.; MORTON, A.; GARCÍA-LÓPEZ, L.; GARCÍA-DEL-PINO, F. Compatibility of entomopathogenic nematodes with natural enemies for horticultural pest control. Biological Control, v.138, p.104050, 2019. https://doi.org/10.1016/j. biocontrol.2019.104050

HESRAN, S.L.;GROOT, T.; KNAPP,M.;BUKOVINSZKY, T.; FORESTIER, T.; DICKE, M. Phenotypic variation in egg survival in the predatory mite Phytoseiulus persimilis under dry conditions. Biological Control, v.130, p.88-94, 2019. https://doi.org/10.1016/j.biocontrol.2018.10.007

JENNIFER, A.; LORENZI, A.; FORAY, V.; HANCE, T. Impact of differences in nutritional quality of wingless and winged aphids on parasitoid fitness. Journal of Experimental Biology, v.221, p.1-11, 2018. https:// doi/10.1242/jeb.185645

LANGHOF, M.; MEYHOFER, R.; POEHLING, H.M.; GATHMANN, A. Measuring the field dispersal of Aphidius colemani (Hymenoptera: Braconidae) Agriculture Ecosystem and Environment, v.107, n.2-3, p.137-143, 2005. https://doi.org/10.1016/j.agee.2004.12.001
MIKAWA, Y.; AIZAWA, M.; UESUGI, R.; OSAKABE, M.; MORI, K.; TOYAMA, M.; SONODA, S. Molecular monitoring of Neoseiulus californicus released from sheltered slow-release sachets for spider mite control in a Japanese pear greenhouse. Experimental and Applied Acarology, v.80, p.203-214, 2020. https://doi.org/10.1007/ s10493-019-00463-4

NEMATI, A.; RIAHI, E.; MOGHADAM, A.K.; GWIAZDOWICZ, D.J.; BAHARI, M.R.; AMINI, P. Comparison of different pollen grains and a factitious prey as food sources for Amblyseius swirskii (Acari: Phytoseiidae). Systematic and Applied Acarology, v.24, n.12, p.24272438, 2019. https://doi.org/10.11158/saa.24.12

NETTING, J.F.; HUNTER, M.S. Ovicide in the whitefly parasitoid, Encarsia formosa. Animal Behavior, v.60, n.2, p.217-226, 2000. https://doi.org/10.1006/anbe.2000.1463

PRADO, S.G.; JANDRICIC, S.E.; FRANK, S.D. Ecological interactions affecting the efficacy of Aphidius colemani in greenhouse crops. Insects, v.6, n.2, p.538-575, 2015. https://doi.org/10.3390/insects6020538

PUNDT, L. 2014. Biological control of two-spotted spider mites. The University of Connecticut. USA. Available at: <http://ipm.uconn.edu/documents/raw2/ html/664.php?aid $=664>$ Accessed on: November $5^{\text {th }}, 2020$.

SARWAR, M. Comparative life history characteristics of the mite predator Neoseiulus cucumeris (Oudemans) (Acari: Phytoseiidae) on mite and pollen diets. International Journal of Pest Management, v.62, n.2, p.140-148, 2016. https://doi.org/10.1080/09670874.2016.1146806

SAITO, T.; BROWNBRIDGE, M. Compatibility of soil-dwelling predators and microbial agents and their efficacy in controlling soil-dwelling stages of western flower thrips Frankliniella occidentalis. Biological Control, v.92, p.92-100, 2016. https://doi.org/10.1016/j. biocontrol.2015.10.003

SHIMODA, T.; KAGAWA, Y.; MORI, K.; NORIHIDE HINOMOTO, N.; HIRAOKA, T.; NAKAJIMA, T. A novel method for protecting slow-release sachets of predatory mites against environmental stresses and increasing predator release to crops. BioControl, v.62, p.495-503, 2017. https://doi.org/10.1007/s10526-017-9800-5

SUMMERFIELD, A. 2019. Biocontrol thriving in Canadian floriculture greenhouses. Greenhouse Canada. Available at: <https://www.greenhousecanada. com/biocontrol-thriving-in-canadian-floriculturegreenhouses-32858/> Accessed on: February 9th, 2021) 
VAN ROERMUND, H.J.W.; VAN LENTEREN J.C. Foraging behaviour of the whitefly parasitoid Encarsia formosa on tomato leaflets. Entomologia experimentalis et applicate, v.76, p.313-324, 1995. https://doi. org/10.1111/j.1570-7458.1995.tb01975.x

VORBURGER, C. Symbiont-conferred resistance to parasitoids in aphids - Challenges for biological control. Biological Control, v.116, p.17-26, 2018. https://doi. org/10.1016/j.biocontrol.2017.02.004
VOSTEEN, I.; BIANCHI, F.J.J.A.; POELMAN, E.H. Adverse weather conditions impede odor-guided foraging of parasitoids and reduce their host-finding success. Agriculture, Ecosystems \& Environment, v.301, p.107066, 2020. https://doi.org/10.1016/j. agee.2020.107066

ZILAHI-BALOGH, G.M.G.; SHIPP, J.L.; CLOUTIER, C.; BRODEUR, J. Influence of light intensity, photoperiod, and temperature on the efficacy of two aphelinid parasitoids of the greenhouse whitefly. Environmental Entomology, v.35, n.3, p.581-589, 2006. https://doi. org/10.1603/0046-225X-35.3.581 